\title{
COMUNICACIÓN VS TECNOLOGÍA
}

\author{
Darwin Toscano Ruíz ${ }^{1}$ \\ Universidad Técnica de Babahoyo (Ecuador) ${ }^{1}$ \\ cepecutb@gmail.com ${ }^{1}$
}

Cómo citar este artículo: Toscano, D. (2015) Comunicación vs Tecnología. CUMBRES, Revista Científica. 1(1) 49 - 55

\section{RESUMEN}

El objetivo de este trabajo está encaminado a profundizar sobre la relación que existe entre la comunicación y la tecnología en los tiempos actuales, teniendo en cuenta su influencia en la educación y en la información. Para esto se consultó una amplia bibliografía publicada en revistas de reconocido prestigio, tesis de doctorados, maestrías, la información recolectada se sometió a un análisis y síntesis, que permitió conformar el artículo. Contiene un análisis conceptual de las principales categorías tratadas, tales como: comunicación y tecnología. Se analizan las dificultades que se presentan con la aplicación de las Tecnologías de la Información y la Comunicación en la educación. Se dispone de una información, donde se pone de manifiesto el rol que han tenido los avances del aprendizaje virtual en el campo educativo y las transformaciones que la misma tecnología ha engendrado en el sistema social.

Palabras clave: Tecnología, comunicación, información, educación.

\section{ABSTRACT}

The objective of this work is aimed at deepening the relationship between communication and technology in modern times, taking into account their influence on education and information. For this an extensive bibliography published in prestigious journals, doctoral theses, masters were consulted, the information collected was subjected to analysis and synthesis, which enabled form the article. It contains a conceptual analysis of the main treated such categories as: communication and technology. The difficulties encountered with the application of information and communications technology in education are analyzed. It has information, where highlights the role that advances have had e-learning in the educational field and the transformations that the same technology has engendered in the social system.

Keywords: Technology, communication, information, education.

\section{INTRODUCCIÓN}

Los problemas de la comunicación han ido adquiriendo un lugar privilegiado en la psicología y en la pedagogía contemporánea. Incluso crece cada día, dentro de la comunidad científica, el consenso de considerar a la comunicación como un principio metodológico primordial al hombre y al desarrollo de su personalidad.

Dentro del ámbito educativo el término comunicación pedagógica (o educativa) también ha venido obteniendo ciudadanía propia, sobre todo en Iberoamérica, al abarcar aquellos procesos interactivos entre educadores y educandos que promueven el perfeccionamiento de la personalidad de ambos.

En las últimas décadas los sistemas de medios de comunicación masiva y de educación han sufrido cambios debido al desarrollo y la difusión de nuevas tecnologías de información y las comunicaciones.

Las tecnologías de la información y la comunicación (TIC) - la unión de los computadores y las comunicaciones- desataron una explosión sin precedentes de formas de comunicarse al comienzo de los años 90. A partir de ahí, la 
Internet pasó de ser un instrumento especializado de la comunidad científica a ser una red de fácil uso que modificó las pautas de interacción social.

En este sentido plantea que diversos son los planteamientos que argumentan la importancia de la incorporación de las TIC a los procesos sustantivos de la Universidad y las transformaciones que se requieren para enfrentar con su utilización la formación de profesionales en la era de la información y el conocimiento (Tunnerman, 1996). Para la Universidad del próximo milenio enfrentar los cambios necesarios para dar respuestas a la diversidad de problemáticas sociales constituye un reto al que podrá enfrentar de forma satisfactoria al incorporar las TIC en toda su actividad.

En lo que se refiere al aspecto docente, (Colom et al., 1988) señala que la presencia de las Tecnologías de la información y de la comunicación ha producido profundos cambios en los medios de enseñanza al incorporar algunos nuevos y cambiar muchos de los métodos y técnicas para la realización de los tradicionales. Estos cambios han influido, además, en la forma de enseñar con los medios, al proporcionar nuevas técnicas que optimizan la formación y ofrecer otros métodos que facilitan el acceso a ésta.

Con relación a la comunicación en sí, (Islas, 2006), indica que estamos ante una transformación del ver y del hacer impulsada por la tecnología. Esto genera nuevos códigos de comunicación que responden a las nuevas realidades de ésta, replanteando los clásicos modelos comunicacionales; imponiéndose la necesidad de reflexionar y profundizar en nuevos elementos de la comunicación.

Al analizar el rol de las tecnologías en educación plantea (Gumucio, 2014), una serie de aspectos, que a nuestro juicio, son de gran importancia para comprender mejor esta problemática, los cuales se relacionan a continuación:

- Los cambios que afectan el papel de la comunicación en la educación no deberían ser ni cosméticos ni instrumentales, sino de enfoque y de proceso. El añadido de nuevas tecnologías sobre un sistema arcaico de educación no es la solución

- Se cree que dotando a las escuelas de computadoras, conectividad de Internet, cámaras de video y estudios de producción radiofónica, se acorta la "brecha" que existe entre una escuela que opera en base a la letra y una escuela que opera en base a la imagen. En ese caso se olvida algo muy obvio: lo que importa no es el libro o la computadora, lo que importa es el aprendizaje de la lectura (del texto o de la imagen, y el puente entre ambos)

- La incorporación de la tecnología es apenas un paso en un nuevo proyecto educativo. La tecnología per se corre el riesgo de perpetuar un sistema decimonónico en lugar de renovarlo, si es que no se renueva a la vez el proceso educativo, basado en competencias emocionales y en experiencias vivenciales

Una educación pertinente que utilice como instrumentos las nuevas tecnologías, tendría sobre todo que crear posibilidades de construir de manera critica el conocimiento, haciendo énfasis en el dialogo y el debate, y en la apreciación crítica de los mensajes audiovisuales y de los propios procesos de comunicación e información.

Kaplún, (1992) refiriéndose a este tema señala que, en su uso más corriente las nuevas Tics ignoran por completo los procesos dialógicos "apelando principalmente a la interactividad (con una máquina) y no a una verdadera interacción (entre personas)", y sobre esto comenta que, el ideal del estudiante aislado y conectado a una máquina niega en los hechos el carácter social del aprendizaje. La navegación solitaria en las autopistas de la información no puede reemplazar el aprendizaje, que es esencialmente social. Considera además que la palabra interactividad es una forma de autismo, y la sustituiría por, la interlocución, la intercomunicación y la interacción, propias del diálogo.

La revolución tecnológica que vive en la humanidad actualmente es debida en buena parte a los avances significativos en las tecnologías de la información y la comunicación. Los grandes cambios que caracterizan esencialmente esta nueva sociedad son: la generalización del uso de las tecnologías, las redes de comunicación, el rápido desenvolvimiento tecnológico y científico y la globalización de la información.

De acuerdo a la situación que presenta este tema según el análisis realizado hasta aquí, se traza como objetivo de este trabajo el de profundizar sobre la relación que existe entre la comunicación y la tecnología en los tiempos actuales, teniendo en cuenta su influencia en la educación y en la información.

\section{MARCO TEÓRICO}

\section{Comunicación}

Según (Nieves, 2008) la palabra Comunicación proviene del latín communis que significa común. Tanto el latín como los 
idiomas romances han conservado el especial significado de un término griego, el de "Koinoonia", que significa a la vez comunicación y comunidad.

Tradicionalmente, la comunicación se ha definido como «el intercambio de sentimientos, opiniones, o cualquier otro tipo de información mediante habla, escritura u otro tipo de señales». Todas las formas de comunicación requieren un emisor, un mensaje y un receptor destinado, pero el receptor no necesita estar presente ni consciente del intento comunicativo por parte del emisor para que el acto de comunicación se realice. En el proceso comunicativo, la información es incluida por el emisor en un paquete y canalizada hacia el receptor a través del medio. Una vez recibido, el receptor decodifica el mensaje y proporciona una respuesta (Berlok, 1969).

Grandes pensadores como Federico Engels, se refirió a este asunto, expresando que la comunicación está estrechamente vinculada con la naturaleza social del hombre y con la evolución de su conciencia. En el proceso de hominización, el trabajo "tenía que contribuir forzosamente a agrupar aun más a los miembros de la sociedad. En resumen, los hombres en formación llegaron a un punto en que tuvieron necesidad de decirse algo los unos a los otros" (Engels, 1973, p.69).

González, (2002) reconoce la comunicación como un mecanismo desarrollador de la personalidad, como condición indispensable y fuente esencial para el desarrollo psíquico.

Al explicar técnicamente el proceso de la comunicación, explica que desde un punto de vista técnico se entiende por comunicación el hecho que un determinado mensaje originado en el punto A llegue a otro punto determinado B, distante del anterior en el espacio o en el tiempo (Islas, 2006). También es un intercambio de ideas y conceptos, por medio del lenguaje en el que damos a conocer historias, experiencias mediante un proceso del emisor y receptor; basado a lo anterior unos de sus elementos principales son:

Código: el código es un sistema de signos y reglas para combinarlos, que por un lado es arbitrario y por otra parte debe de estar organizado de antemano.

Canal: el proceso de comunicación que emplea ese código precisa de un canal para la transmisión de las señales. El Canal sería el medio físico a través del cual se transmite la comunicación.

Ej.: El aire en el caso de la voz y las ondas Hertzianas, en el caso de la televisión. La radiocomunicación es un sistema de telecomunicación que se realiza a través de ondas de radio $\mathrm{u}$ ondas hertzianas.

En tercer lugar debemos considerar el emisor. Es la persona que se encarga de transmitir el mensaje. Esta persona elige y selecciona los signos que le convienen, es decir, realiza un proceso de codificación; codifica el mensaje.

El receptor será aquella persona a quien va dirigido el mensaje; realiza un proceso inverso al del emisor, ya que descifra e interpreta los signos elegidos por el emisor; es decir, descodifica el mensaje.

Naturalmente tiene que haber algo que comunicar, un contenido y un proceso que con sus aspectos previos y sus consecuencias motive el mensaje.

Las circunstancias que rodean un hecho de comunicación se denominan contexto situacional (situación); contexto en que se transmite el mensaje y que contribuye a su significado.

Consecuencias de la comunicación: es positiva, cuando el receptor de la misma, interpreta exactamente lo que el emisor le envió; esto quiere decir que utilizaron el mismo canal de comunicación y es el objetivo primordial de la misma. Es negativa, cuando el receptor utiliza un canal de comunicación diferente al del emisor, y es muy frecuente que suceda este tipo de comunicación distorsionada, cuando el receptor no está anclado en la misma línea de comunicación; Es conveniente que el emisor utilice un lenguaje claro y de acuerdo al nivel cultural del receptor para que el mensaje sea descifrado correctamente.

La comunicación está presente en nuestras vida y permite afianzar conocimientos a lo largo de esta, todos tenemos diferentes formas de comunicarnos pero siempre tenemos un objetivo claro con lo que queremos decir, es por eso que las tecnologías han dado paso a una nueva forma de comunicarnos ya sea escrita, oral, aunque algunos han adoptado estas como una herramienta de uso diario.

\section{Tecnología}

Según el Glosario de la (UNESCO, 1999), Tecnología es el conjunto de conocimientos científicos y empíricos para alcanzar un resultado práctico: un producto, un proceso de fabricación, una técnica, un servicio, una metodología.

Según (Ciapuscio, 1999) la tecnología es el conjunto de conocimientos técnicos, científicamente ordenados, que permiten diseñar y crear bienes y servicios que facilitan la adaptación al medio ambiente y satisfacer tanto las necesidades esenciales como los deseos de la humanidad. 
Es una palabra de origen griego, $\tau \varepsilon \chi \vee o \lambda o y i ́ a$, formada por téchnē ( $\tau \dot{x} \chi \vee \eta$, arte, técnica u oficio, que puede ser traducido como destreza) y logía (入oyía, el estudio de algo).

La tecnología es concebida como conocimiento aplicado en casi todas las actividades humanas, conocimientos que han desempañado un rol importante en los logros materiales y culturales en la evolución de la sociedad. Sin la tecnología no podrían realizarse algunas actividades humanas, pues es ella la que establece el "como" se ejecuta la actividad. Y, no puede realizarse acción humana alguna sin cierto acervo de conocimientos empíricos o racionales acerca del mundo físico, biológico o social (Tapias, 2000).

La revolución de la ciencia y la tecnología (en particular, las tecnologías de la información y la comunicación) ha transformado profundamente, no sólo el sistema productivo, sino la estructura social en los países industrializados. Este proceso repercute con fuerza en los países en desarrollo y, por el momento, se traduce en un gran desconcierto con respecto a las políticas que corresponde adoptar (Albornoz, 2001).

Las tecnologías usan, en general, métodos diferentes del científico. Los métodos difieren según se trate de tecnologías de producción artesanal o industrial de artefactos, de prestación de servicios, de realización u organización de tareas de cualquier tipo.

Los medios de comunicación se han valido desde un principio de la tecnología para hacer valer su sentido informativo. La tecnología hace que la comunicación de masas crezca cada día más, desde el punto de vista de que las fronteras van siendo eliminadas, las personas pueden acceder cada vez de una forma más rápida y accesible a la información, aunque quizás no de forma más veraz.

\section{Tecnologías de la información y la comunicación}

Según (Malbernat, 2010) las tecnologías de la información y la comunicación (TIC), a veces denominadas nuevas tecnologías de la información y la comunicación (NTIC) son un concepto muy asociado al de informática. Si se entiende esta última como el conjunto de recursos, procedimientos y técnicas usadas en el procesamiento, almacenamiento y transmisión de información, esta definición se ha matizado de la mano de las TIC, pues en la actualidad no basta con hablar de una computadora cuando se hace referencia al procesamiento de la información.

Internet puede formar parte de ese procesamiento que, quizás, se realice de manera distribuida y remota. Y al ha- blar de procesamiento remoto, además de incorporar el concepto de telecomunicación, se puede estar haciendo referencia a un dispositivo muy distinto a lo que tradicionalmente se entiende por computadora pues podría llevarse a cabo, por ejemplo, con un teléfono móvil o una computadora ultra-portátil, con capacidad de operar en red mediante comunicación inalámbrica y con cada vez más prestaciones, facilidades y rendimiento.

Al explicar la composición de estas tecnologías, (Sarramona, 1998) señala que en la denominación de las tecnologías de la comunicación y la información, se engloban aquellas que permiten la adquisición, producción, almacenamiento, tratamiento, comunicación, registro y representación de datos e informaciones obtenidas en señales de naturaleza acústica, óptica o electromagnética.

Estas tecnologías aparecen como un proceso de convergencia de distintas áreas de conocimiento y aplicación (electrónica, informática, telecomunicación automática) que, si bien hasta la década de los comienzos de los sesenta se desarrollaban con cierta independencia, hoy día están estrechamente relacionadas entre sí.

Para (Aguilar, 2003) las tecnologías de la información y la comunicación, conocidas con las siglas TIC, son el conjunto de medios (radio, televisión y telefonía convencional) de comunicación y las aplicaciones de información que permiten la captura, producción, almacenamiento, tratamiento, y presentación de informaciones en forma de voz, imágenes y datos contenidos en señales de naturaleza acústica, óptica o electromagnética.

Las TIC incluyen la electrónica como tecnología base que soporta el desarrollo de las telecomunicaciones, la informática y el audiovisual.

Según (Castañeda, 2003), las TIC son el resultado de las posibilidades creadas por la humanidad en torno a la digitalización de datos, productos, servicios y procesos, y de su transportación a través de diferentes medios, a grandes distancias y en pequeños intervalos de tiempo, de forma confiable, y con relaciones costo-beneficio nunca antes alcanzadas por el hombre.

Al analizar los efectos de las TIC, (Delors, 1996) expresa que, a pesar de sus ventajas comunicativas también pueden separar. Estrechan la comunicación entre quienes las utilizan, pero excluyen a quienes no.

Es evidente que las políticas educativas mucho tienen que decir en relación a evitar, o al menos, compensar estas 
desigualdades en el acceso a la información y el conocimiento en la mayoría de los países del tercer mundo.

\section{Tecnologías de la información y la comunicación en la educación}

Los rápidos progresos que traen consigo las nuevas tecnologías seguirán modificando las formas de elaboración, adquisición y transmisión de los conocimientos. Permitirán, además, aprovechar las posibilidades que brindan para la educación y la enseñanza al mejorar la manera de producir, organizar, difundir y controlar el saber, y de acceder al mismo. Por tanto, las tecnologías constituyen un instrumento, una herramienta importante para ser aplicada con éxito en los procesos educativos, ya sean en la educación formal regular o la educación especial (Serradas, 2004).

Actualmente las tecnologías de la información y la comunicación están sufriendo un desarrollo vertiginoso, esto está afectando a prácticamente todos los campos de nuestra sociedad, y la educación no es una excepción. Esas tecnologías se presentan cada vez más como una necesidad en el contexto de sociedad donde los rápidos cambios, el aumento de los conocimientos y las demandas de una educación de alto nivel constantemente actualizada se convierten en una exigencia permanente. (Rosario, 2005)

Valdés, (2000) al tratar los retos que establecen las nuevas tecnologías de la información y la comunicación a la práctica docente actual, plantea que deben ser incorporadas a un proceso renovado y renovador de enseñanza-aprendizaje, donde se empleen en beneficio del desarrollo de competencias que permitan formar individuos para un aprendizaje a lo largo de toda la vida. Del mismo modo, agrega que deben ser utilizadas en beneficio de la atención de individualidades, sus necesidades, conocimientos previos, motivaciones que den un carácter significativo al aprendizaje, como proceso activo de construcción de conocimientos, desarrollo de capacidades y sentimientos que generen una actitud responsable hacia sí mismo y hacia los demás.

La introducción de las TIC en el proceso de enseñanza-aprendizaje como contenido y como medio de enseñanza, como cultura y como recurso social, y como reto a todos sus actores, es una realidad y una necesidad social impuesta por el desarrollo tecnológico de la sociedad, ante las potencialidades de esta tecnología, las relaciones costo/beneficio alcanzadas por ella para muchas esferas de la vida y por la dinámica que le ha impuesto a muchas de estas esferas, sin que se vean con precisión aún muchos de sus límites (Castañeda, 2003).
Las escuelas deben cambiar como proyecto educativo, no como infraestructura. No basta aterrizar computadoras y conectividad con Internet, no bastan las cámaras de video y los estudios de radio. Se necesita una escuela que promueva procesos de aprendizajes reflexivos y basados en la experiencia, relevantes socialmente, es decir, insertos en una realidad social más amplia.

El hecho de que la computadora y las demás tecnologías de información puedan servir para la educación es innegable. Esto sin embargo no significa que las tecnologías de información sean principalmente tecnologías educativas. Por el contrario, su aparición en la sociedad ha estado motivada por múltiples eventos, unos técnicos, otros económicos, otros políticos. Su vinculación con los procesos educativos no ha sido ni es automática. En el contexto moderno de los países capitalistas su vinculación además no nace de una necesidad educativa sino de una económica principalmente. Montero

La formación a través del uso de las nuevas tecnologías plantea un nuevo horizonte para el mundo educativo, libre de barreras físicas y temporales, adaptado a las necesidades de los alumnos y basado en la interacción y en el aprendizaje cooperativo

El desarrollo constante de las TIC, con las que se abren posibilidades de soluciones prácticas a problemas esenciales de la educación a distancia está exigiendo nuevas interpretaciones de conceptos como: distancia, comunicación interpersonal, aprendizaje, u otros, que se encuentran en estudio y desarrollo (Collazo, 2004)

\section{Información y educación}

El desarrollo de Internet ha significado que la información esté ahora en muchos sitios. Antes la información estaba concentrada, la transmitía la familia, los maestros, los libros. La escuela y la universidad eran los ámbitos que concentraban el conocimiento.

Rojas, (2009) explica que en la actualidad vivimos en un mundo rodeado de información, en donde los diferentes estilos de vida de las sociedades desarrolladas y los sistemas económicos, están apoyadas por herramientas tales como las TIC (tecnologías de la información y comunicación), son los principales motores generadores de información. La sociedad actual se encuentra en crecimiento constante, dando origen así a ingentes cantidades de documentos, lo cual nos permite creer que estamos construyendo a escala planetaria una sociedad de la información, entendiendo por dicha sociedad a aquella en la cual 
la creación, distribución y manipulación de la información, forman parte trascendente de las actividades culturales y económicas, tomando en cuenta que la tecnología es una de las claves técnicas de la Sociedad de la Información.

A fines de 1980 la UNICEF adoptó el término "tercer canal" para referirse a todos los instrumentos disponibles y canales de información, comunicación y acción social (que) pueden ser usados para ayudar a transmitir los conocimientos esenciales e informar y educar a la población en asuntos sociales. Asumiendo la educación formal y no-formal como los otros dos canales educativos (UNICEF, 1990).

La Conferencia Mundial sobre Educación para Todos, cifró grandes esperanzas en este "tercer canal" para alcanzar las seis metas de educación básica para todos a cumplirse para el año 2000.

Llegado el 2000, no obstante, e incumplidas las metas, éstas se redujeron y los plazos se aplazaron hasta el 2015.

El "tercer canal", antes pensado como un canal amplio compartido por tecnologías tradicionales y modernas, desapareció de las metas educativas y se redujo a las TIC.

En un análisis sobre esta problemática, (Torres, 2000) señala al respecto algunos nudos críticos sobre los cuales se debe reflexionar y debatir:

- La educación en la sociedad de la información es un enunciado problemático, difícil de resignificar, atrapado en dos términos: educación (cuando lo que se busca el aprendizaje, el aprender a aprender) e información (cuando el objetivo es trascender el dato, conocer, comprender, aprender, crear).

- Indiferenciación entre información y conocimiento, entre sociedad de la información y Sociedad de la Comunicación, y uso de ambos como si fuesen intercambiables, con acento sobre la información más que sobre la comunicación.

Indiferenciación entre información y educación, capacitación y formación, educación y aprendizaje. De estas indiferenciaciones y simplificaciones surgen atribuciones a las TIC que no corresponden y aún pocas exploraciones sistemáticas acerca del potencial informativo, comunicacional y propiamente educativo/formativo de las TIC y sus variados usos.

- Las modernas TIC colocadas en el centro de la información y la comunicación, desplazando a las TIC tradicionales y a instituciones claves como la familia, la comunidad, el sistema escolar, los medios masivos de comunicación, la biblioteca, el lugar de trabajo, etc.
- Las TIC (y las propias nociones de información y comunicación) reducidas a computadora e Internet. Además, el apelativo de "modernas" para calificar a las TIC es relativo; hay otras tecnologías modernas, otras lo fueron antes y éstas dejarán de serlo pronto.

Área, (2003) al referirse a la preparación que deben tener los profesores, plantea que los docentes deben poseer un concepto crítico de las características principales de la revolución informática, así como de algunas ideas relativas al futuro de la denominada "sociedad de la información", de sus ventajas e inconvenientes y de las posibilidades de acceso a los medios tecnológicos en igualdad de oportunidades para todos.

- Nos sirve como resumen lo planteado por Kofi Annan en el discurso inaugural, en la Cumbre Mundial sobre la Sociedad de la Información (CMSI), que expresó: "Las tecnologías de la información y la comunicación no son ninguna panacea ni fórmula mágica, pero pueden mejorar la vida de todos los habitantes del planeta. Se dispone de herramientas para llegar a los Objetivos de Desarrollo del Milenio, de instrumentos que harán avanzar la causa de la libertad y la democracia y de los medios necesarios para propagar los conocimientos y facilitar la comprensión mutua" (Kofi Annan, 2003).

\section{CONCLUSIONES}

1. Como resultado de este trabajo, se dispone de una información, donde se pone de manifiesto el rol que han tenido los avances de la tecnología en la comunicación y la información, que han incidido notablemente en el campo educativo y transformaciones que la misma tecnología ha engendrado en el sistema social.

2. Es consenso de la mayoría de los autores consultados, que las tecnologías de la Información y las Comunicaciones, deben considerarse un medio y no un fin en sí mismas y que el profesor al emplearlas debe crear posibilidades de construir de manera critica el conocimiento, haciendo énfasis en el dialogo y el debate, y en la apreciación crítica de los mensajes audiovisuales y de los propios procesos de comunicación e información.

3. Se dispone de una información, donde se pone de manifiesto el rol que han tenido los avances de lasTecnologías de la Información y lasComunicaciones, en el campo educativo y transformaciones que la misma tecnología ha engendrado en el sistema social. 


\section{REFERENCIAS BIBLIOGRÁFICAS}

- Aguilar, J. (2003). Historia de la Sociedad de la Información. Hacia la sociedad del Conocimiento" en Revolución tecnológica. Alicante: Universidad de Alicante.

- Albornoz, M. (2001). Política Científica y Tecnológica Una visión desde América Latina. Revista Iberoamericana de Ciencia, Tecnología, Sociedad e Innovación.

- ÁREA, M. (2003). Sociedad de la información y analfabetismo tecnológico: nuevos retos para la educación de adultos. Sevilla: Universidad de Sevilla. (http://tecnologiaedu.us.es/bibliovir.htm.2005/06).

- Berlok, K. (1969). "El proceso de la comunicación (introducción a la teoría y la práctica). Argentina: El Ateneo.

- Castañeda, Á. (2003). El papel de las tecnologías de la información y las comunicaciones (TICS) en el proceso de enseñanza aprendizaje a comienzos del siglo XXI. La Habana, Cuba: Preparación pedagógica integral para profesores universitarios.

- Ciapuscio, H. (1999). Nosotros \& la tecnología. Buenos Aires, Argentina: Editorial Ágora, ISBN 9789879623558.

- Collazo, R. (2004). Una concepción teórico - metodológica para la producción de cursos a distancia basados en el uso de las tecnologías de la información y las comunicaciones. La Habana, Cuba: Tesis en opción al título de Doctor en Ciencias de la Educación. Instituto Superior Politécnico José Antonio Echeverría.

- Colom, A., Salinas, J. y Sureda, J. (1988). Tecnología y medios educativos. Madrid, España: Cincel Kapelusz.

- Delors, J. (1996). La educación encierra un tesoro. Madrid, España: Informe a la UNESCO de la Comisión Internacional sobre la Educación para el siglo XXI.

- Engels, (1973). Papel del trabajo en el proceso de transformación del mono en hombre, p. 66 79. Obras escogi $\neg$ das, tomo III. Editorial Progreso, Moscú, 613 p.

- Glosario de la UNESCO (1999). Revista El Correo.

- González, J. (2002). Autoexpresión y Comunicación interpersonal en la organización. La Habana, Cuba: Facultad de Comunicación. U. Habana. Grupo Cambio Humano. Impresión Ligera.

- Gumucio, A. (2014). Comunicación y Educación: Una deuda recíproca. La Hojarasca, Alianza de escritores y periodistas. Disponible en (http://www.escritoresyperiodistas.com/NUMERO31/NUMERO31.htm).

- Islas, O. (2006). La era McLuhan, parteaguas teórico en las ciencias de la comunicación. Monterrey, México: Tecnológico de Monterrey. En Revista Mexicana de Comunicación.

- Kaplún, M. (1992). "A la educación por la comunicación: la práctica de la comunicación educativa". Santiago de Chile: UNESCO, OREALC.
- Kofi, A. (2003). Discurso inaugural de la primera fase de la WSIS. Ginebra: Organización de las Naciones Unidas.

- Malbernat, L. (2010). Tecnologías educativas e innovación en la Universidad. LaCapitalmdp.com.

- Nieves, F. (2008). Comunicación Organizacional. En Conceptos de comunicación. http://www.gestiopolis. com.

- Rojas, Y. (2009). Reflexiones en torno a la Sociedad de la información y el conocimiento. Madrid, España: Revista de Estudios Literarios. Universidad Complutense de Madrid.

- Rosario, J. (2005). La Tecnología de la Información y la Comunicación (TIC). Su uso como Herramienta para el Fortalecimiento y el Desarrollo de la Educación Virtual. Disponible en el archivo del Observatorio para la CiberSociedad en http://www.cibersociedad.net/archivo/articulo.php?art=218.

- Sarramona, J. (1998). El profesor de la Universidad Autónoma de Barcelona. Madrid, España: Revista de Girona No 189, ISSN 0211-2663.

- Serradas, M. (2004). Las nuevas tecnologías como recurso en la respuestaeducativa a los niños hospitalizados. Caracas, Venezuela: Teleeducación de aulas hospitalarias. Revista de Pedagogía, 25 (74), ISSN 0798-9792.

- Tapias, H. (2000). Gestión tecnológica y desarrollo tecnológico. Antioquia, Colombia: Revista de la Facultad de Ingeniería. Universidad de Antioquía. Disponible en: http//jaibaina.udea.edu.co/producciones/Heberto/gestión.

- Torres, R. (2000). Formación docente: clave de la reforma educativa. En Nuevas formas de aprender y enseñar. Santiago de Chile: UNESCO.

- Tunnerman, C. (1996). Conferencia Regional sobre políticas y estrategias para la transformación de la educación superior en América Latina. La Habana, Cuba: CRESAL. Conferencia Introductoria.

- UNICEF (1990). Children and Development in the 1990s. New York: A UNICEF Sourcebook, on the ocasión of the World Summit for Children.

- Valdés, N. (2000). Reto de las NTI y las Comunicaciones al Diseño Curricular y la práctica docente actual. Revista Contexto Educativo. № 7. Disponible en: http://contexto-educativo.com.ar 